\title{
High Resolution Characterization on Point Defects in (Pr,Al) Implanted $\mathrm{SrTiO}_{3}$
}

\author{
Guozhen Zhu, Andrew P. Knights*, Gianluigi A. Botton
}

Canadian Centre for Electron Microscopy and Department of Materials Science and Engineering, McMaster University, 1280 Main St. W., ON, Canada, L8S 4M1

*Centre for Emerging Devices Technology and Department of Engineering Physics, McMaster University, 1280 Main St. W., ON, Canada, L8S 4M1

$\mathrm{SrTiO}_{3}$ doped with $\mathrm{Pr}$ (STO:Pr) is of special interest because it shows red cathodoluminescence with an efficiency as high as $0.4 \mathrm{~lm} / \mathrm{W}$ at an anode voltage of $400 \mathrm{~V}$ and an acceptable efficiency at low voltages even below 10V. [1] In addition, by co-doping with a small amount of selected elements such as $\mathrm{Al}, \mathrm{SrTiO}_{3}: \mathrm{Pr}$ becomes a bright phosphor used for field-emission displays and vacuum fluorescent displays. [2] A few possible explanations have been proposed for this increased efficiency. It has been proposed [1] that $\mathrm{Al}^{3+}$ ions preferentially locate around $\mathrm{Pr}^{3+}$ ions, in order to compensate the charge difference between the $\operatorname{Pr}^{3+}$ ions and the host ions, which, as a consequence, improves the crystallinity and changes the crystal field around $\mathrm{Pr}^{3+}$ ions. On the other hand, Kutty and Nag [3] attributed such effect to the formation of secondary nano-particles and the associated interfacial defects. However, due to the lack of direct experimental evidence, no clear understanding of rare earth luminescence in this system is available. Here we present first observations of STO:Pr co-doped with $\mathrm{Al}$ in order to elucidate the nanoscale structure of these materials.

$\mathrm{Pr}^{+}$ions were implanted in (001) $\mathrm{SrTiO}_{3}$ single crystals at an energy of $35 \mathrm{keV}$ and a dose of $7 \times 10^{13} \mathrm{~cm}^{-2}$ at room temperature (RT). $\mathrm{Al}^{+}$ions were implanted subsequently at $15 \mathrm{keV}$ to a dose of $3.8 \times 10^{14} \mathrm{~cm}^{-2}$ at RT. To remove the damage caused by the implantation processes, annealing treatments at $400^{\circ} \mathrm{C}, 700^{\circ} \mathrm{C}$ and $800^{\circ} \mathrm{C}$ for $5-180$ mins were carried out after implantation. Photoluminescence of as-implanted and annealed crystals were investigated using a $\mathrm{He}-\mathrm{Cd}$ pump laser of $325 \mathrm{~nm}$ wavelength at RT. Detailed structural characterization was carried out with highangle annular dark-field (HAADF) scanning transmission electron microscopy (STEM) with an aberration-corrected FEI Titan 80-300 Cubed microscope.

The emission peaks in the photoluminescence (PL) spectra, related to the intra-4f transitions of $\mathrm{Pr}^{3+}$ ions, include one broad peak of $\sim 610 \mathrm{~nm}$ wavelength and several narrow atomic-like peaks. The intensity of the broad peak is greatly enhanced with increasing annealing temperature. In the asimplanted crystals, we observe a 25 30nm thick amorphous layer and 7-15nm thick crystalline layer with a high density of defects (Fig. 1a). Following annealing, the layer with a high-density of defects re-crystallizes at $400^{\circ} \mathrm{C}$ with this effect being more pronounced at higher annealing temperatures. In the crystal annealed at $800^{\circ} \mathrm{C}$, the amorphous layer is fully re-crystallized. Cube-shaped nanoclusters of about $0.8-2.4 \mathrm{~nm}$ size were observed in the implanted regions (Fig. 1b) appearing with a reduced intensity with respect to the STO background. We detected no clusters with higher intensity than the background in HAADF images suggesting that the Pr ions are uniformly distributed rather than in clusters.

In order to understand the site-preference of the $\mathrm{Pr}^{3+}$ ions and assess whether a solid solution of STO with Pr ions is present, we carried out visibility tests using multi-slice simulations [4] to identify the imaging and sample requirements for the detection of single atoms in the sample. Our simulations suggest that directly imaging $\operatorname{Pr}(\mathrm{Z}=59)$ in crystalline $\mathrm{SrTiO}_{3}$ could only be achieved in samples with a thickness less than $5 \mathrm{~nm}$ (Fig.2) The visibility of Pr in the Al clusters within STO is currently being investigated to assess the relationship between the $\mathrm{Pr}$ and $\mathrm{Al}$ distributions in the crystals.[5] 


\section{References}

[1] Toki, H., et al., Proc. Third Int. Display Workshops 2, 1996.

[2] Yamanoto, H., et al., J. Luminesc., 100 (2002): 325

[3] Kutty, T.R.N., et al., J. Mater. Chem. 13(2003): 2271

[4] Kirkland, E. J., Advanced Computing in Electron Microscopy, Plenum, New York, 1998.

[5] This research was supported by NSERC. The aid of Doris Stevanovic (CEDT, McMaster) for the implantation is gratefully acknowledged.

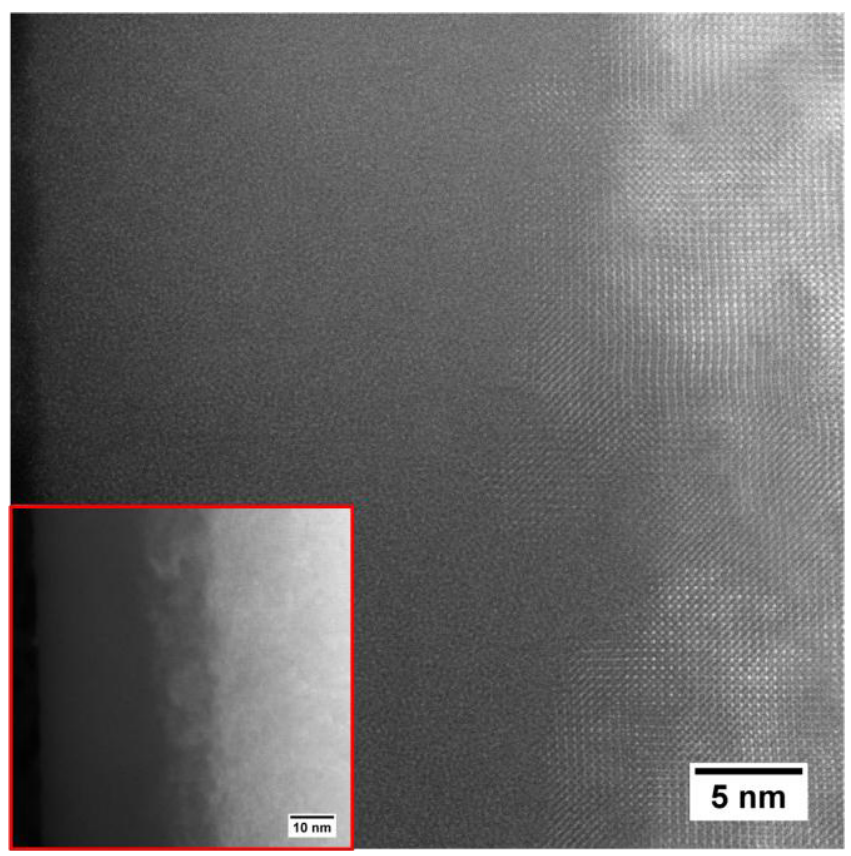

(a)

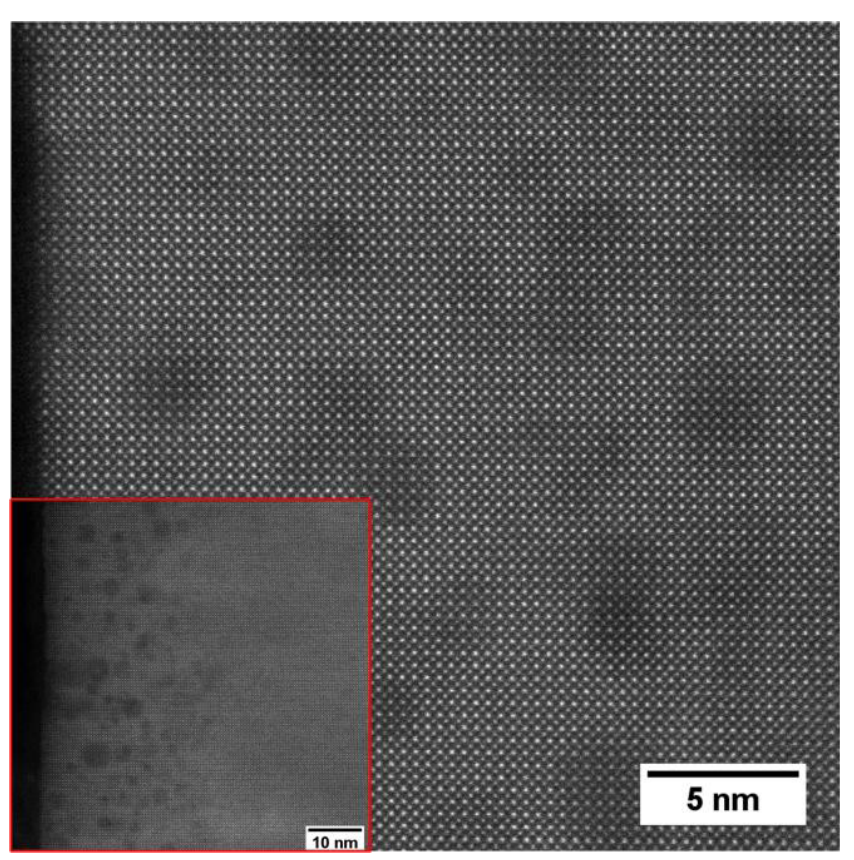

(b)

FIG. 1. STEM-HAADF images of as-implanted (a) and annealed (b) $\mathrm{SrTiO}_{3}$ single crystals annealed at $800^{\circ} \mathrm{C}$ for $3 \mathrm{hrs}$. Cross-section wedge specimens were prepared by the low-angle polishing followed by the ion milling with $0.25-1.5 \mathrm{keV} \mathrm{Ar}^{+}$ions. In the inserts, low magnification images showing the field of view and location of the damaged layer.

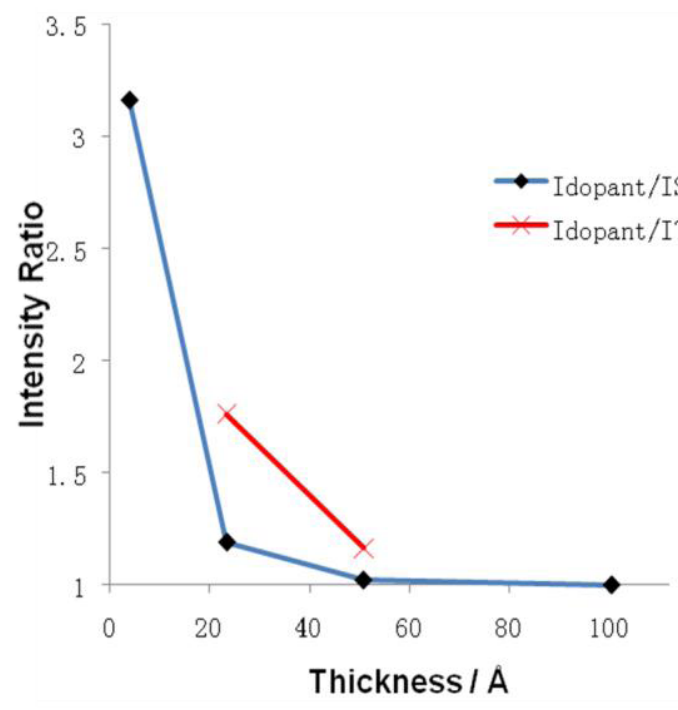

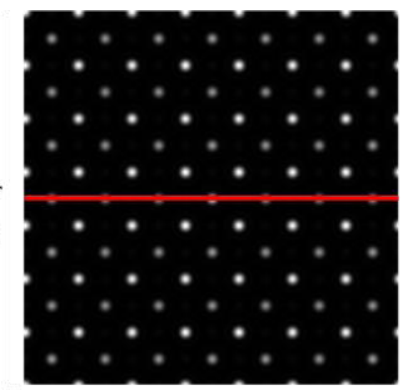

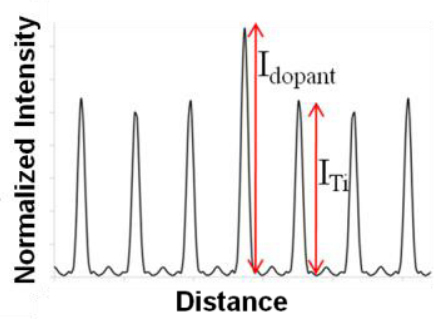

FIG. 2. The visibility of $\mathrm{Pr}$ dopants in $\mathrm{SrTiO}_{3}$. The visibility is defined as the intensity ratio between the atomic column that includes the dopant with its neighboring atomic column. In the inset, the HAADF image calculated with a single $\mathrm{Pr}$ impurity at the centre of the supercell with the related line scan showing the intensity. The Pr atom is on a substitutional Ti site (red curve) and $\mathrm{Sr}$ site (blue curve). 